\title{
Gradhiva
}

GRADHIV

Revue d'anthropologie et d'histoire des arts

26 | 2017

En croire ses sens

\section{Anouk Cohen, Fabriquer le livre au Maroc}

Paris, Karthala/IISMM, coll. « Terres et gens d'islam », 2016

\section{Dinah Ribard}

\section{CpenEdition}

Journals

Édition électronique

URL : http://journals.openedition.org/gradhiva/3488

DOI : 10.4000/gradhiva.3488

ISSN : 1760-849X

Éditeur

Musée du quai Branly Jacques Chirac

Édition imprimée

Date de publication : 6 décembre 2017

Pagination : 226-227

ISBN : 978-2-35744-096-8

ISSN : 0764-8928

Référence électronique

Dinah Ribard, «Anouk Cohen, Fabriquer le livre au Maroc », Gradhiva [En ligne], 26 | 2017, mis en ligne le 06 décembre 2017, consulté le 24 septembre 2020. URL : http://journals.openedition.org/gradhiva/ 3488 ; DOI : https://doi.org/10.4000/gradhiva.3488

Ce document a été généré automatiquement le 24 septembre 2020.

(c) musée du quai Branly 


\section{Anouk Cohen, Fabriquer le livre au}

\section{Maroc}

Paris, Karthala/IISMM, coll. « Terres et gens d'islam », 2016

Dinah Ribard

\section{RÉFÉRENCE}

Anouk Cohen, Fabriquer le livre au Maroc. Paris, Karthala/IISMM, coll. « Terres et gens d'islam », 2016, 411 p.

\section{Coll. Terres et gens d'islam}

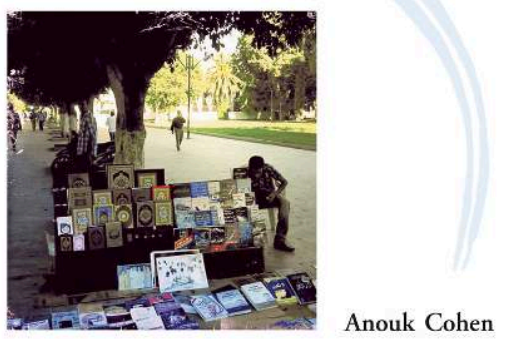

\section{Fabriquer le livre} au Maroc

IISMM - KARTHALA 
1 Anouk Cohen souhaitait étudier le changement à l'œuvre dans la société marocaine d'aujourd'hui en s'intéressant à la presse et à la littérature féminines, toutes deux d'apparition récente. Elle a rencontré un phénomène plus large : l'apparition du livre marocain. Aujourd'hui, on peut acheter au Maroc des ouvrages fabriqués au Maroc aussi bien qu'en Égypte, au Liban ou en France. Depuis les années 1990, des auteurs marocains, hommes et femmes, écrivent des livres concernant le Maroc ou leur propre expérience qui trouvent à être imprimés, vendus et lus dans leur pays. De nouveaux titres de presse sont apparus, caractérisés, à l'exemple de la presse féminine, par le souci de parler aux Marocains de la société dans laquelle ils vivent. Les maisons d'édition sont certes fragiles et se soutiennent souvent par la coédition, c'est-à-dire la diffusion sur le marché marocain, à prix réduit, d'ouvrages édités ailleurs, qu'elles modifient à la marge et se chargent de distribuer; mais alors qu'elles n'étaient qu'une poignée, elles se sont multipliées dans les villes marocaines, à Rabat et à Casablanca en particulier. Ainsi des témoignages sur la répression politique au temps de Hassan II ontils pu être publiés, d'abord dans la presse, puis par des éditeurs marocains, et connaître un succès de librairie important. De même, le Coran est devenu un «objet de consommation " courante, grâce à l'activité d'éditeurs-libraires spécialisés de Casablanca qui conçoivent et font fabriquer en grand nombre par leurs partenaires égyptiens ou libanais des exemplaires destinés aux Marocains, qu'ils peuvent vendre très peu cher. L'enquête d'Anouk Cohen permet de reconnaître un fait dans cet ensemble de déplacements, et d'en comprendre l'importance : que signifie, pour une société d'aujourd'hui, fabriquer ses propres livres, lorsque ce qui se lisait jusque-là venait d'ailleurs?

2 La question donne un sens plus complexe, par exemple, à une de ces mesures chiffrées qui semblent montrer l'évolution d'un pays en développement : la mesure de la baisse de l'illettrisme dans le royaume - bien moins important chez les plus jeunes que chez les plus âgés, chez les garçons que chez les filles, dans les villes que dans les campagnes. Plus de gens qui savent lire, au Maroc, peuvent aujourd'hui se procurer de la lecture en achetant librement des marchandises imprimées (voire photocopiées, moins chères encore) devenues plus abordables, mais aussi et d'abord achetables, dans l'anonymat de l'échange commercial. Le Coran, vendu à des milliers d'exemplaires chaque mois - ce qui signifie qu'un habitant de Casablanca, par exemple, en acquiert en moyenne un tous les quatre ans, voire plus souvent - n'est plus seulement entre les mains des hommes chargés d'en permettre la lecture. Il s'offre en cadeau, il se transporte avec soi, dans des formats de poche dont les plus orthodoxes désapprouvent l'existence. De nombreux ouvrages pratiques, vendus comme le Livre saint dans la rue et par les buralistes aussi bien qu'en librairie, font perdre au volume imprimé son inaccessibilité prestigieuse. Les livres qui nourrissent la critique sociale ou la contestation politique ne circulent plus seulement de main en main, invisiblement, après avoir été achetés en France et apportés au Maroc. Ce n'est donc pas seulement que plus de gens savent lire : c'est qu'ils lisent autrement, et par là même autre chose.

3 Le Maroc d'aujourd'hui fabrique ses propres livres écrits en arabe et ses propres livres écrits en français, ainsi qu'un petit nombre d'ouvrages en langue berbère (trouvables, note Anouk Cohen, chez les vendeurs de rue plutôt qu'en librairie, car le pouvoir n'est pas favorable à leur diffusion, et les librairies sont plus contrôlables). Ces différents types de livres marocains ne sont pas toujours achetables dans les mêmes lieux, et rarement par les mêmes personnes, peu nombreuses étant celles qui lisent aussi 
aisément dans une langue que dans l'autre. Ils ne font pas les mêmes lecteurs, puisque ce sont aussi les lecteurs qui sont fabriqués par les écrits qui leur parviennent. Le bilinguisme du livre marocain a ainsi pour effet de répartir les transformations évoquées plus haut entre des lectorats partiellement différents. Anouk Cohen montre toute la complexité du phénomène en étudiant le scandale soulevé par la publication d'un dossier consacré aux plaisanteries que les Marocains s'échangent sur le sexe, la politique et la religion par un magazine, rédigé dans le dialecte marocain, et non dans l'arabe classique ou standard utilisé normalement à l'écrit. Ce n'est pas, en effet, la publication proprement dite qui a fait scandale : le même dossier avait précédemment été publié en français dans un magazine possédé par le même groupe de presse. C'est la lisibilité autre des plaisanteries imprimées, la création, autour de ces mots d'esprit, d'un lectorat non restreint aux gens capables de lire l'arabe écrit. Militer en acte, comme le fait l'un des titres arabophones les plus vendus à l'époque de l'enquête (2005-2010), pour donner au dialecte marocain le statut de langue écrite officielle, c'est bouleverser un équilibre linguistique profondément politique. Ce que ne font pas, pour leur part, les témoignages de victimes de la répression politique sous Hassan II, publiés librement dans la même période, et même utilisés depuis lors comme archives par la commission créée par Mohammed VI pour faire un sort au passé récent du pays. Ils sont en effet, pour l'essentiel, publiés en français, tandis que des éditeurs arabophones font paraître des livres d'histoire muets, eux, sur cette part de l'histoire du royaume. Cela ne veut pas du tout dire que leur mise sur le marché n'a pas eu d'impact, mais simplement que ses acteurs ont respecté les règles de distribution de l'impact des livres sur les lecteurs en fonction de la langue qu'ils lisent. Le numéro sur les blagues marocaines a quant à lui été retiré de la vente. Mais par cet acte de censure, le pouvoir politique a finalement protégé les journalistes des conséquences d'un scandale qu'il n'avait pas suscité, et qui le contraignait même à agir au nom de sa fonction de garant du respect de la religion et de la morale publique. En réalité, tout le monde, Palais aussi bien qu'éditeurs-libraires et auteurs, a intérêt à l'existence de deux lectorats séparés par leur langue. Signe de démocratisation, la fabrication d'écrits critiques est désormais bienvenue - et souvent subventionnée d'une manière ou d'une autre par des institutions françaises, autres actrices de ce jeu de normes -, pourvu qu'il soit acquis que les lecteurs arabophones en sont préservés, l'écart entre langue parlée et langue écrite étant censé assurer que le peuple marocain demeurera de toute façon à distance de ce que les livres peuvent lui faire. Reste qu'on peut raconter ce qu'on a lu à qui ne lit pas, que quelques poèmes en arabe évoquent bel et bien l'expérience d'anciens prisonniers politiques - la poésie étant l'un des genres privilégiés par l'imprimé arabe -, qu'un magazine en dialecte a trouvé beaucoup d'acheteurs et que la littérature islamique récente peut fabriquer elle aussi des lecteurs critiques.

4 Anouk Cohen a demandé à un historien du livre à l'époque moderne, Roger Chartier, de préfacer son ouvrage. C'est que cette enquête d'ethnologie, menée en travaillant pendant plusieurs mois chez différents éditeurs et libraires francophones et arabophones, en observant la manière dont les gens achètent, en interrogeant un grand nombre d'auteurs, d'ouvriers et ouvrières du livre, de vendeurs de rue et de bouquinistes des souks, de lecteurs, est, comme il le note, une mise à l'épreuve passionnante des propositions de l'histoire du livre. Depuis L'Apparition du livre, l'ouvrage pionnier cosigné par Lucien Febvre et Henri-Jean Martin (1958), ce sont toutes les transformations sociales et culturelles occasionnées par le passage du manuscrit à l'imprimé, par la fabrication des livres que l'auteure s'est donné pour 
objectif de mesurer et de comprendre. Bien des hypothèses pourront se trouver enrichies par l'enquête d'Anouk Cohen sur les conditions et les conséquences de l'apparition très contemporaine du livre marocain dans un monde déjà plein de livres.

5 Fabriquer le livre au Maroc, le titre judicieusement choisi pour suggérer les enjeux de cette enquête, ne rend pourtant pas tout à fait compte de son ampleur. Toutes les étapes de la fabrication, de l'écriture à la réalisation matérielle des exemplaires destinés à la vente, sont effectivement abordées, ce qui est déjà remarquable : il est rare, en réalité, que le travail des écrivains et celui des éditeurs (y compris pour obtenir des subventions ou des marchés scolaires) et des employés d'imprimerie soient embrassés dans une même étude. Ici, les réflexions de différentes auteures sur l'histoire familiale, intime et sociale de leur entrée dans l'écriture et sur le sens qu'a pour elles le choix de l'arabe ou du français répondent à celles des éditeurs-libraires sur la manière dont ils mêlent concurrence et coopération avec des confrères chez qui ils passent souvent de longues heures à prendre le thé, ou sur les couleurs et le graphisme des couvertures des ouvrages qu'ils mettent en vente. Toutes sont instructives de plusieurs manières. Ainsi voit-on avec les éditeurs de Casablanca - et grâce à quelques-unes des nombreuses et utiles photographies insérées par Anouk Cohen - que les livres fabriqués en France sont habillés pour être vendus en France, avec des couleurs plus claires que celles, plus vives, que portent les livres marocains dont on suit dans d'autres chapitres la confection. La démarche permet de saisir comment agit sur les tâches de tous les travailleurs du livre et sur ceux et celles qui les exercent la contrainte absolue de fabriquer à bas coût pour vendre à bas prix. Mais plusieurs chapitres sont également consacrés aux différentes manières qu'ont les habitants des villes marocaines de se procurer de quoi lire, dans les librairies et les kiosques ou aux étalages posés à même le sol des rues par des vendeurs quelquefois illettrés qui se servent des couleurs des couvertures pour classer leur marchandise, en louant des ouvrages pour un temps, en se les prêtant ou en achetant des exemplaires photocopiés, ou encore en se rendant dans des bibliothèques. D'autres chapitres s'intéressent au rangement des livres dans les logements, aux endroits où l'on peut voir les gens lire à Casablanca et à Rabat, aux façons de lire en public et à la lecture privée. Bref, ce sont toutes les formes de présence du livre qui ont retenu l'attention d'Anouk Cohen. D'une immense richesse, d'une lecture passionnante, son ouvrage ne suscite qu'un regret: parfois convenue, affaiblie par la référence à des théorisations si générales des faits culturels qu'elles valent pour les cas les plus différents, l'analyse n'a pas toujours la même acuité que la remarquable description qui la rend possible.

\section{AUTEURS}

\section{DINAH RIBARD}

dinah.ribard@ehess.fr 